\title{
Identificación de factores que afectan la satisfacción del alumnado de educación sobre la tutorización en su formación práctica. Un estudio con ANOVA
}

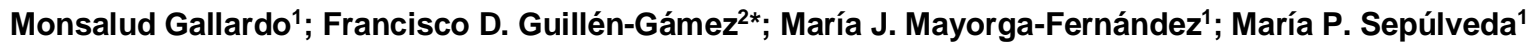 \\ (1) Departamento de Didáctica y Organización Escolar, Universidad de Málaga. Málaga-España \\ (correo-e: monsalud@uma.es; mjmayorga@uma.es; mdsepulveda@uma.es) \\ (2) Departamento de Didáctica y Organización Escolar, Universidad de Almería. Almería-España. \\ (correo-e: dguillen@ual.es)
}

*Autor a quien debe ser dirigida la correspondencia

Recibido Dic. 4, 2019; Aceptado Feb. 1, 2020; Versión final Feb. 25, 2020, Publicado Jun. 2020

\begin{abstract}
Resumen
El objetivo del presente trabajo es indagar en la satisfacción del alumnado respecto a la labor de tutorización desarrollada por el tutor/a académico y profesional durante las prácticas externas en los grados de educación, así como identificar las variables demográficas y académicas que afectan significativamente a su satisfacción. Para ello, se plantea un estudio con una metodología ex post facto descriptiva e inferencial con una técnica de encuesta. Los resultados han demostrado que no existen diferencias significativas respecto a género en relación a la satisfacción del alumnado con la labor del tutor/a académico, mientras que sí existen en lo referente al tutor/a profesional, estando las mujeres más satisfechas con dicha labor. Aunque la satisfacción general respecto a la tutorización es media-alta, se puede concluir que es fundamental que las instituciones de educación superior hagan una apuesta política y destinen más recursos a optimizar la labor tutorizadora.
\end{abstract}

\section{Identifying factors that affect education students' satisfaction with tutoring in their training practice. A study with ANOVA}

\begin{abstract}
The aim of this research is to analyze education student's approval of academic and non-academic professional tutors in the student's external practices. This study also identifies demographic and academic variables that significantly affect student satisfaction with tutoring. A survey study is performed using an ex post facto descriptive and inferential methodology. The results show that the overall satisfaction regarding tutoring is medium-high. There are no significant differences between gender and student satisfaction with the tutoring performed by academic tutors. However, female students are more satisfied with the tutoring of non-academic professional tutors. It can be concluded that it is essential for higher education institutions to improve tutoring jobs by making a political commitment to tutoring and by allocating more resources.
\end{abstract}

Keywords: external practice; gender; tutoring; higher education 


\section{INTRODUCCIÓN}

Actualmente las instituciones educativas no pueden seguir formando a ciudadanos/as como lo hacían en el siglo XIX, sino que tienen que prepararlos para que sepan vivir y hacer frente a las demandas y retos que se les plantean en la sociedad del siglo XXI (Martínez et al., 2012). Desde este posicionamiento, las Facultades de Ciencias de la Educación, de acuerdo a los requerimientos del Espacio Europeo de Educación Superior (EEES), han otorgado un mayor protagonismo al alumnado en el proceso de enseñanza-aprendizaje, focalizándose la enseñanza en los aprendizajes (Casillas et al., 2017), es decir, el desarrollo de competencias en el alumnado se ha convertido en el núcleo central de los planes de estudio.

En esta línea, para implementar un currículo que fomente el desarrollo de competencias debe estar definido por un modelo educativo que apueste por la formación integral de los profesionales (Alonso-García, et al., 2018), basado en una visión interrelacionada entre saber, saber hacer y saber ser (Olmedo et al., 2016). Esto implica la concepción de un aprendizaje interdisciplinar para la vida, la adquisición de un aprendizaje relevante que desarrolle las cualidades humanas fundamentales. Más aún, supone la construcción de modalidades de conocimiento más complejas, contextualizadas, que integren conceptos, habilidades y destrezas profesionales, así como actitudes personales que permitan a los estudiantes ocupar su lugar en la sociedad. Esto conlleva que los procesos de enseñanza-aprendizaje estén abiertos a nuevas concepciones epistemológicas donde la relación teoría-práctica se co-implican en un movimiento permanente de mutuo enriquecimiento.

En este marco, las prácticas externas se convierten en uno de los pilares fundamentales de cualquier plan de estudio universitario, para mejorar la autoeficacia de los futuros maestros/as (Martins et al., 2015). Las prácticas externas, por tanto, se pueden entender como el conjunto de actividades curriculares y externas, diseñadas por las distintas Universidades, donde se tienen presentes las voces y las aportaciones de los profesionales de los diferentes ámbitos de actuación, y que son desarrolladas por los estudiantes universitarios durante su formación inicial en entornos laborales (Gairín et al., 2019). Las prácticas deben proporcionar al alumnado una visión global de su futura actividad profesional, así como de los diferentes ámbitos donde la van a desarrollar, convirtiéndose en un medio para integrar al alumnado en un marco de aprendizaje real y donde va a desempeñar de un rol profesional (Valverde, 2016; Sulistiyo et al., 2017; Teng, 2017; Holtz y Gnambs, 2017), que les va a permitir aprender desde la experiencia en contextos reales (Yangin y Gungor, 2018). Asimismo, constituyen un contexto donde el alumnado pone en práctica gran parte de las competencias que ha adquirido a lo largo de su formación inicial, convirtiéndose en espacios formativos claves para la reconstrucción de su pensamiento práctico (Vicente, 2012; Mayorga et al., 2017).

En este proceso formativo hay que destacar dos figuras fundamentales: el tutor/a académico (profesor/a universitario) y el tutor/a profesional (del centro de prácticas), quienes, de forma coordinada, son los principales responsables de promover esa reconstrucción del pensamiento práctico, así como el desarrollo de competencias personales y profesionales en el alumnado. El presente trabajo, por tanto, pretende: por un lado, conocer el nivel de satisfacción del alumnado, de último curso, respecto a la tutorización que reciben por parte del tutor/a académico y del tutor/a profesional, durante la realización de la última asignatura de prácticas antes de obtener el Grado que están cursando; y por otro lado, conocer si diferentes variables académicas y personales influyen de manera significativa sobre el grado de satisfacción del alumnado en relación a la tutorización.

\section{OTROS ANTECEDENTES}

Para comprender mejor el tema de estudio es necesario analizar: i) las prácticas como eje de los grados de educación, y ii) la coordinación durante la tutorización.

\section{Las prácticas como eje de los grados de educación}

Las prácticas externas, en la actualidad, están tomando cada vez más relevancia en la ordenación y estructuración universitaria, puesto que son, especialmente en las titulaciones relacionadas con la educación, una oportunidad única para que el alumnado diseñe y experimente, en contextos profesionales, propuestas de actuación a partir del análisis realizado, y de los aprendizajes adquiridos a través de las diferentes materias. Así mismo, posibilita que el alumnado tome conciencia de las debilidades y fortalezas de sus propuestas y puedan rediseñarlas. Este periodo formativo, por tanto, constituye una ocasión para profundizar en el contexto profesional, y reflexionar sobre las acciones desarrolladas en el mismo, así como sobre las decisiones adoptadas. En definitiva, el sentido de las prácticas externas es fomentar el aprendizaje holístico del alumnado, es decir, el desarrollo de competencias básicas fundamentales y lograr, consecuentemente, un aprendizaje pre-profesional clave en su formación inicial (Cramer, 2016). Para ello, los planes de estudio deben otorgar a las prácticas externas el lugar que les corresponde en el currículo, 
siendo necesario revisar su organización temporal, puesto que Liesa y Vived (2010) encontraron que, de los 27 países que conforman Europa, más del $50 \%$ apuestan por una mayor presencia temporal de las prácticas en los planes de estudio, duplicando la permanencia del alumnado en los centros, pasándose de 30 a 60 créditos; asimismo, las prácticas deben tener un claro y coherente plan de tutorización o seguimiento (Overton, 2018), siendo imprescindible la coordinación entre los agentes implicados en el proceso de enseñanza-aprendizaje.

Ahora bien, para reconstruir el pensamiento práctico y adquirir las competencias profesionales no basta con incrementar los créditos de formación práctica en los planes de estudios, sino que hay que fortalecer el proceso de tutorización en dicho período, y establecer una mayor relación entre los tutores/as académicos y los profesionales mediante reuniones consensuadas y plasmadas en un calendario escolar, que puedan dar lugar a guías o planes coordinados para la mejora de las prácticas y la creación de espacios de reflexión interinstitucionales, tal y como ponen de manifiesto Sánchez et al. (2011) en un estudio realizado en la Facultad de Educación y Humanidades de Ceuta (Universidad de Granada). Llegados a este punto, es necesario plantear el debate los roles y funciones que deben desempeñar tanto los tutores/as académicos como los tutores/as profesionales durante el desarrollo de las prácticas.

\section{La coordinación durante la tutorización}

Durante las prácticas externas, el alumnado tiene la oportunidad de aprender haciendo en un contexto profesional bajo una directa supervisión, de hecho, se puede afirmar que el éxito de las prácticas depende, en gran medida, del apoyo y asesoramiento que reciba por parte de sus tutores/as: orientación, supervisión, asesoramiento y feedback, tanto por parte del tutor/a académico como del tutor/a profesional (Nicol et al., 2014). En este sentido, es necesario hacer una mención especial al tutor/a profesional, pues constituye el nexo entre el mundo universitario y el mundo profesional (Martínez y Raposo, 2011). Las funciones del tutor/a profesional son numerosas, pero se pueden concretar en las siguientes: acoger al estudiante en prácticas, integrarlo en la dinámica del centro, informarle, facilitarle materiales y conocimiento del contexto, planificar y organizar sus actuaciones, analizar conjuntamente con ellos las intervenciones realizadas así como las decisiones que ha adoptado durante el desarrollo de las mismas, implicar al resto de profesionales del centro o institución, establecer un clima que favorezca el aprendizaje, estar disponible, mantener una relación abierta y de confianza, provocar la reflexión del estudiante, realizar un seguimiento y evaluarlo, etc. (Sánchez et al., 2011). Por su parte, el tutor/a académico también ha de realizar el seguimiento del alumnado, evaluarlo y provocar procesos de reflexión, que faciliten en éste la reconstrucción de su pensamiento práctico. Para ello, el tutor/a académico ha de promover en todo momento la interacción teoría y práctica, adentrarse en las teorías declaradas y proclamadas del alumnado, ayudarles a que expliciten sus planteamientos, etc. Como se puede apreciar, se comparten responsabilidades y, por ello, se hace indispensable que se intensifiquen las relaciones y la coordinación entre ambos profesionales para trabajar en un único proyecto o plan de trabajo.

A nivel nacional, en el territorio español, los estudios analizados ponen de manifiesto la importancia que tiene optimizar la labor tutorizadora tanto de los tutores/as profesionales como de los tutores/as académicos (Martínez y Raposo, 2011) de cara al desarrollo de competencias por parte de los estudiantes. Al respecto, Cid et al. (2011), en 137 estudios cualitativos basados en las prácticas externas, concluyeron que, para los estudiantes en prácticas, la tutorización les proporcionaba: apoyo para su desarrollo profesional, ayuda para la realización de aprendizajes fundamentados en la reflexión, así como ideas nuevas sobre la enseñanza, es decir, estrategias de innovación, que favorecen el desarrollo de competencias. Por su parte, Pinto (2019) a través de un estudio con 113 estudiantes (futuros maestros/as de Educación Primaria) procedentes de la Universidad de Salamanca (España), destacó que los estudiantes estaban muy satisfechos con la experiencia adquirida en las prácticas escolares, teniendo la oportunidad de observar el contexto real, demostrar sus habilidades de enseñanza y mejorar las competencias adquiridas. En líneas similares, López et al. (2012) con 432 estudiantes del Grado de Psicología de la Universidad de Salamanca (España), también resaltaron la importancia de la tutorización, considerando un $62,30 \%$ del alumnado encuestado que dicha tutorización era buena y/o muy buena. Vallejo et al. (2019) con una muestra de 537 estudiantes de los Grados de Educación Infantil y Primaria de la Universidad de Jaén (España) obtuvieron una elevada satisfacción de dichos estudiantes en relación a la tutorización, siendo ligeramente superior el nivel de satisfacción en relación al tutor/a profesional en comparación con el tutor/a académico. Además, no se encontraron diferencias significativas respecto al género, ni respecto al grado educativo que estaban cursando.

Por otro lado, a nivel internacional también se ha dedicado especial interés al estudio de las prácticas. García et al. (2018) en su estudio de corte cualitativo plantearon una propuesta formativa dirigida a 10 tutores de prácticas del Instituto Preuniversitario Vocacional de Ciencias Exactas "Federico Engels" de Pinar del Río (Cuba). Los resultados mostraron que en el proceso de tutorización había escasa planificación de 
las acciones, homogeneización en el trato con el alumnado, así como una escasa formación previa recibida desde la institución. En México, Chan-Pavon et al. (2018) realizaron un estudio para conocer el nivel de satisfacción de 74 estudiantes de Ingeniería Química respecto a sus prácticas profesionales, los resultados mostraron un alto nivel de satisfacción, pero no se analizaron las labores de tutorización del tutor/a académico ni profesional.

Por otro lado, en Australia, McGraw \& Davis (2017) realizaron una investigación cualitativa con 2 escuelas rurales, para conocer en qué medida el contexto influía en la tutorización profesional. Los resultados mostraron que efectivamente un buen trabajo en equipo y una buena comunicación en los centros educativos favorecía una retroalimentación positiva con el alumnado en prácticas, resultados coincidentes con los de Maddamsetti (2018), puesto que es necesario fomentar en mayor medida la colaboración entre tutores profesionales, escuelas y universidades. En este mismo contexto, Izadinia (2016) realizó un estudio cualitativo en la Universidad Occidental Australiana, donde analizó las percepciones tanto de los estudiantes como de tutores académicos respecto a las relaciones de tutorización, en total participaron 8 personas en el estudio. Aunque, tanto tutores como estudiantes resaltaron la importancia de la comunicación y el feedback, los estudiantes encontraron deficiencias en relación a que la tutorización no les había ayudado lo suficiente a construir su identidad profesional. En la misma línea, Yuan (2016) realizó un estudio de casos con dos estudiantes de Magisterio de Inglés procedentes de una Universidad de China, para conocer en qué medida la tutorización académica y profesional contribuía a la construcción de la identidad de estudiantes de magisterio, el estudio concluyó que ambas tutorizaciones influían significativamente en la construcción de dicha identidad, tanto en sentido positivo como negativo, y que es necesario promover la efectividad de la tutorización.

Rodríguez-Loera y Onrubia (2019), por su parte, concluyeron que para que los futuros docentes pudieran durante las prácticas reconstruir su pensamiento práctico era necesario una mayor ayuda y retroalimentación entre tutores profesionales y académicos, así como una mayor unificación de los criterios de evaluación de las prácticas. Sin embargo, según Helleve \& Ulvik (2019), en un estudio realizado con 16 tutores profesionales de diferentes colegios de secundaria de Noruega, mostraron que los tutores/as no tenían claras cuáles eran las competencias de los tutores académicos, y que era necesario un mayor conocimiento de las competencias que debían poner en práctica, así como una mayor comunicación entre ellos. Es más, Ruffinelli et al. (2020) llevaron a cabo con 27 estudiantes de Chile una formación fundamentada en prácticas reflexivas junto a sus respectivos tutores académicos y profesionales, en dicho estudio se concluyó que la creación de entornos para la reflexión conjunta potenció el aprendizaje mutuo y la colaboración Universidad-Escuela. Melesse (2014), tras analizar las experiencias de 120 estudiantes (futuros maestros/as de educación), 36 profesores universitarios (tutores/as académicos) y 66 tutores/as profesionales de prácticas, determinó que, aunque las prácticas ayudaron a los estudiantes a identificar sus puntos débiles y fuertes en contextos reales, poner en práctica la teoría adquirida en la universidad y desarrollar confianza en sí mismos, los resultados revelaron que existían problemas de coordinación y comunicación entre los agentes implicados, provocando una ausencia de seguimiento cercano y apoyo en los estudiantes, dichos resultados son similares a los obtenidos por Nguyen (2017).

A pesar de la importancia que tiene la labor tutorizadora, en la actualidad, se aprecia que no recibe la atención ni valoración que debiera (Liesa y Vived, 2010), encontrándose algunas limitaciones y deficiencias. Todo ello conlleva que, si los tutores/as no realizan un análisis del contenido de las prácticas, en profundidad, poco o nada impactarán los resultados en el aprendizaje, produciéndose poca conexión entre teoría y práctica. Por tanto, es fundamental que los tutores/as reflexionen en profundidad sobre el sentido y contenido de la formación práctica, así como en el modo de facilitar la interacción entre teoría-práctica tan pretendida en las Facultades y, por otro lado, sobre las estrategias más adecuadas para provocar sinergias entre las escuelas y las facultades (Gairín et al., 2019).

Teniendo en consideración los estudios previos que se han llevado a cabo en relación a las prácticas externas y al nivel de satisfacción del alumnado sobre las labores de tutorización tanto del tutor/a académico como del profesional, el presente trabajo contribuye a dar un paso más en este tipo de estudios, ya que profundiza en como diferentes variables demográficas y académicas afectan a dicho nivel de satisfacción, ya que apenas existen estudios enfocados a este propósito.

Por ello que los objetivos principales de este trabajo han sido: 1) conocer la satisfacción del alumnado respecto a las labores de tutorización, tanto del tutor/a académico, como del tutor/a profesional; y 2) conocer si el género de los estudiantes y diferentes variables académicas (vía de acceso a la etapa universitaria, lugar de realización de las prácticas externas, calificaciones del alumnado en prácticas externas previas, diferentes grados de educación) influyen de manera significativa sobre el grado de satisfacción del alumnado en relación a la tutorización de sus prácticas. 


\section{METODOLOGÍA}

A continuación, se presenta el diseño metodológico del estudio, el cual se divide en tres secciones: i) diseño; ii) participantes; e iii) instrumento.

\section{Diseño}

En este estudio, se ha empleado un diseño de tipo descriptivo ex post facto, a través de encuesta. Los datos se obtuvieron a través de una encuesta de lápiz y papel a las personas que habían sido seleccionadas para el estudio, durante el segundo semestre del curso 2017/2018. Previo a la aplicación del cuestionario, se les explicó a dicho alumnado el objetivo del estudio y la opción de participar de forma voluntaria; además, en el encabezado del cuestionario se garantizaba el anonimato en el tratamiento de los datos.

\section{Participantes}

La muestra se ha seleccionado mediante un muestreo aleatorio simple sin reposición, estando constituida por un total de 251 estudiantes, matriculados en el $4^{\circ}$ curso de los diferentes Grados que se imparten en la Facultad de Educación de la Universidad de Málaga: Pedagogía ( $\mathrm{N}=45)$; Educación Primaria ( $\mathrm{N}=93)$; Educación Social $(\mathrm{N}=25)$; y Educación Infantil $(\mathrm{N}=88)$. En la Tabla 1 se observa la distribución de la muestra en función del género, grado educativo y lugar de realización de las prácticas externas.

Tabla 1: Distribución de los participantes

\begin{tabular}{|l|l|l|l|l|l|l|l|l|l|}
\hline Grado educativo & \multicolumn{2}{|l|}{ Pedagogía } & \multicolumn{2}{l|}{ Edu. Primaria } & \multicolumn{2}{l|}{ Edu. Social } & \multicolumn{2}{l|}{ Edu. Infantil } \\
\hline $\begin{array}{l}\text { Género \Lugar de } \\
\text { prácticas }\end{array}$ & $\begin{array}{l}\text { Centro } \\
\text { educativo }\end{array}$ & $\begin{array}{l}\text { Institución } \\
\text { social }\end{array}$ & $\begin{array}{l}\text { Centro } \\
\text { educativo }\end{array}$ & $\begin{array}{l}\text { Institución } \\
\text { social }\end{array}$ & $\begin{array}{l}\text { Centro } \\
\text { educativo }\end{array}$ & $\begin{array}{l}\text { Institución } \\
\text { social }\end{array}$ & $\begin{array}{l}\text { Centro } \\
\text { educativo }\end{array}$ & $\begin{array}{l}\text { Institución } \\
\text { social }\end{array}$ \\
\hline Femenino & 15 & 16 & 49 & 10 & 5 & 11 & 59 & 10 & 175 \\
\hline Masculino & 3 & 11 & 22 & 12 & 4 & 5 & 15 & 4 & 76 \\
\hline Total & 45 & 93 & & 25 & 88 & & 251 \\
\hline
\end{tabular}

\section{Instrumento}

Para la recogida de datos se ha empleado una de las dimensiones del cuestionario elaborado por González y Hevia (2011), readaptado por Mayorga et al. (2017), llamado: "Agentes implicados en la formación práctica". La primera sección incluía variables demográficas y académicas como el género, la edad, el curso académico matriculado, entre otras. El instrumento estaba compuesto por dos dimensiones. La primera subdimensión estaba enfocada a las percepciones del alumnado sobre su tutor/a académico, incluyendo 8 ítems. La segunda sub-dimensión estaba enfocada en las percepciones del alumnado sobre el tutor/a del centro de prácticas (tutor/a profesional), incluyendo 9 ítems. En total del instrumento, estaba compuesto por estos 17 ítems donde se utilizó una escala Likert de 5 puntos de valoración, donde el valor uno representaba a "Totalmente en desacuerdo" y 5 "Totalmente de acuerdo".

\section{RESULTADOS Y DISCUSIÓN}

La presentación de los resultados se divide en tres secciones: la primera sección está enfocada en analizar las propiedades psicométricas del instrumento a través de la fiabilidad y validez de dicho instrumento; en la segunda sección se pretende conocer el nivel de satisfacción de los estudiantes en relación a las labores de tutorización tanto del tutor/a académico como del tutor/a profesional; la tercera sección se centra en conocer si diferentes variables académicas y personales afectan al nivel de satisfacción del alumnado en relación a las labores de tutorización de los agentes implicados en su formación práctica.

\section{Análisis de la fiabilidad y validez del instrumento.}

Se ha analizado las propiedades psicométricas del instrumento utilizando la fiabilidad por consistencia interna ( $\alpha$ de Cronbach), donde los parámetros obtenidos fueron: $\alpha=0.890$ (total del instrumento), $\alpha=0.933$ (tutor académico), $\alpha=0.947$ (tutor profesional). Respecto a los análisis de la varianza, se ha realizado un análisis factorial exploratorio (AFE) a través del análisis por eje principales con rotación oblimin. Los resultados obtenidos con el índice de adecuación muestral (KMO) es 0.923 y la prueba de esfericidad de Bartlet es significativa ( $\mathrm{sig}=0.000$ ), indicando que la matriz de correlaciones supera las condiciones para la realización de este análisis. Se ha encontrado que los dos factores explican un $70.81 \%$ de la varianza total. El factor 1 engloba los ítems sobre el tutor/a profesional, los cuales explican el $38.38 \%$ de la varianza total; y el factor 2 engloba los ítems sobre el tutor/a académico, los cuales explican el 32.43 de la varianza total. 
Los pesos factoriales obtenidos por cada ítem son altos en el factor 1 , oscilando entre 0.519 y 0.923 ; mientras que en el factor 2 oscilan entre 0.578 y 0.906 . Respecto a la validez del instrumento, un análisis factorial confirmatorio (CFA) fue llevado a cabo, reportando un buen ajuste del modelo: valor para el ajuste estadístico discrepancia mínima / grados de libertad (CMIN / DF, 2.86); índice de ajuste incremental (IFI, 0.94); índice de ajuste comparativo (CFI, 0.94); y la raíz cuadrada media del error de aproximación (RMSEA, 0.08). Además, se ha observado cómo no existen correlaciones entre los diferentes factores latentes (Tutor/a académico y Tutor/a profesional, $r=0.005$ ) al ser factores sobre diferentes agentes implicados. A continuación, en la Figura 1 se muestra la estructura del CFA.

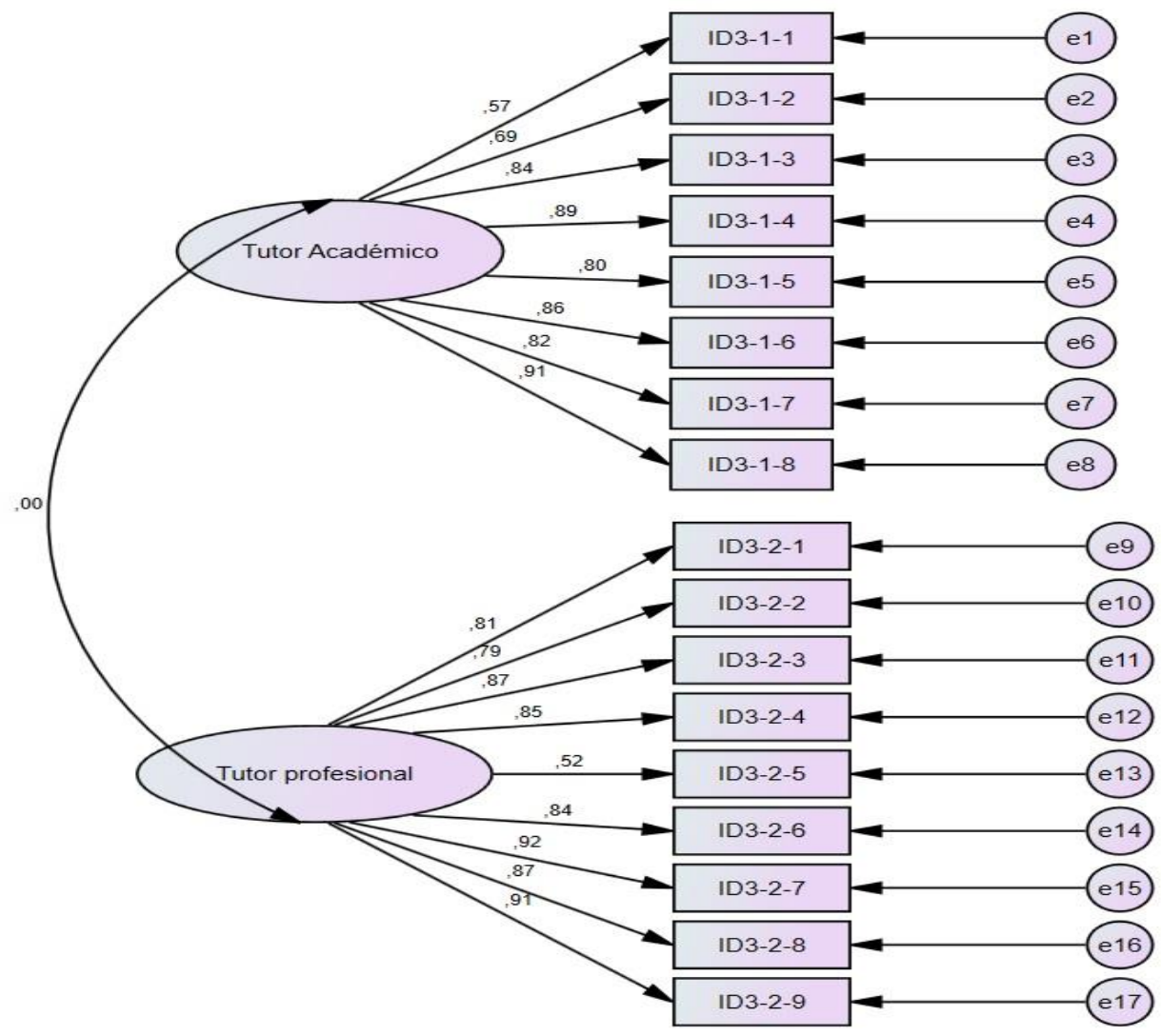

Fig. 1: Estructura del CFA

\section{Análisis de las percepciones del alumnado}

La Tabla 2 muestra la media (M) y desviación típica (DT) sobre la satisfacción del alumnado respecto a su tutor/a académico de la institución universitaria. La percepción general del alumnado sobre las labores del tutor/a académico es medio-alta $(\mathrm{M}=3.38 \pm 0.99)$. Específicamente, se observa cómo los ítems acerca de las labores del tutor/a sobre proporcionar información para el acceso a las prácticas (ID311, $M=3.71 \pm 1.16$ ), así como la obligatoriedad de asistir a tutorías entre el alumnado-tutor/a (ID315, M=3.72 \pm 1.13 ) son aquellos ítems en los que el alumnado percibe una mayor satisfacción. Por el contrario, la percepción del alumnado acerca de la comunicación y coordinación entre el tutor/a académico y profesional es baja (ID312, $\mathrm{M}=2.77 \pm 1.45)$.

Tabla 2: Resultados en la percepción del alumnado sobre su tutor/a académico

\begin{tabular}{|c|c|c|c|}
\hline Ítem & Descripción & M & $D T$ \\
\hline ID311 & Previo a la llegada al centro, el tutor proporciona información para el acceso: carta presentación, referencias. & 3.71 & 1.16 \\
\hline ID312 & El tutor académico mantiene una buena comunicación y coordinación con el tutor profesional & 2.77 & 1.45 \\
\hline ID313 & Asesora adecuadamente en el diseño del Plan de Trabajo, Propuesta de Intervención y elaboración del Portafolios & 3.28 & 1.22 \\
\hline ID314 & Efectúa un seguimiento correcto de las prácticas, manteniendo un feedback adecuado & 3.26 & 1.19 \\
\hline ID315 & Las tutorías obligatorias permiten el intercambio de información entre alumnado y tutor/a & 3.72 & 1.13 \\
\hline ID316 & El tutor académico actúa como elemento de apoyo en la resolución de problemas durante el desarrollo de las prácticas & 3.56 & 1.20 \\
\hline ID317 & Lleva a cabo con eficacia las tutorías individuales & 3.37 & 1.23 \\
\hline ID318 & En general, el tutor de la Facultad orienta satisfactoriamente las prácticas & 3.41 & 1.24 \\
\hline Total & Satisfacción general del alumnado sobre su tutor académico & 3.38 & 0.99 \\
\hline
\end{tabular}


En la Tabla 3, se muestra la satisfacción del alumnado sobre sus tutores/as profesionales en los centros de prácticas. Se observa cómo la satisfacción general del alumnado es medio-alto ( $M=3.79 \pm 0.99$ ). Específicamente, se aprecia cómo los ítems sobre la acogida del tutor/a al alumnado en el centro de prácticas (ID321, M=4.17 \pm 1.00 ) y una actitud cercana hacia el estudiante en el desarrollo de sus prácticas escolares (ID328, M=4.11 \pm 1.11 ) son aquellos ítems en los que el alumnado percibe una mayor satisfacción sobre su tutor/a profesional. Aunque es cierto que el alumnado percibe una buena satisfacción en cada uno de los ítems de la sub-dimensión, el ítem con peor valoración es aquel donde el tutor/a profesional debe comunicarse y coordinarse con el tutor/a académico (ID325, $M=2.66 \pm 1.27$ ).

Tabla 3: Resultados en la percepción del alumnado sobre su tutor/a profesional

\begin{tabular}{|l|l|l|l|}
\hline Ítem & Descripción & $M$ & $D T$ \\
\hline ID321 & Realiza una adecuada acogida en el Centro/Institución & 4.17 & 1.00 \\
\hline ID322 & Explica y describe el funcionamiento y programas del Centro/Institución & 3.87 & 1.11 \\
\hline ID323 & Asesora y orienta al alumnado en sus actividades día a día & 3.90 & 1.17 \\
\hline ID324 & Proporciona los recursos necesarios para la realización de actividades durante las prácticas & 3.89 & 1.23 \\
\hline ID325 & Se comunica habitualmente con el/la tutor/a académico & 2.66 & 1.27 \\
\hline ID326 & Potencia la participación autónoma del estudiante: innovaciones, resolución de conflictos & 3.70 & 1.28 \\
\hline ID327 & Desarrolla de forma eficaz la atención al estudiante en prácticas & 3.82 & 1.20 \\
\hline ID328 & Demuestra una actitud personal cercana hacia el estudiante que realiza las prácticas & 4.11 & 1.11 \\
\hline ID329 & Demuestra una actitud personal cercana hacia el estudiante que realiza las prácticas & 3.98 & 1.19 \\
\hline Total & Satisfacción general del alumnado sobre su tutor académico & 3.79 & 0.99 \\
\hline
\end{tabular}

Influencia de diferentes variables (tutoría académica)

Con el fin de conocer si diferentes variables académicas y demográficas afectan a la satisfacción del alumnado sobre las labores de tutorización de sus tutores/as académicos y tutores/as profesionales, se llevó a cabo dos ANOVA univariantes. El primero se enfocó en conocer si dichas variables independientes (VI) afectan a la satisfacción del alumnado sobre su tutor/a académico (VD 1); el segundo en conocer si dichas VI afectan a la satisfacción del alumnado sobre su tutor/a profesional (VD 2). La Tabla 4 muestra las diferentes variables utilizadas en este estudio, detallando el nivel de medida.

Tabla 4: Definición de las variables

\begin{tabular}{|l|l|l|}
\hline Descripción & Tipo & Nivel de Medida \\
\hline Satisfacción sobre el tutor académico & VD1 & Escala \\
\hline Satisfacción sobre el tutor profesional & $\mathrm{VD2}$ & Escala \\
\hline Género & $\mathrm{VI}$ & Nominal (masculino / femenino) \\
\hline Forma de acceso a los estudios universitarios & $\mathrm{VI}$ & Nominal (pruebas de acceso a la universidad/otros) \\
\hline Grado educativo que cursa actualmente el alumnado & $\mathrm{VI}$ & Nominal (educaciónsocial/educaciónprimaria/educacióninfantil/pedagogía) \\
\hline Lugar de realización de las prácticas & $\mathrm{VI}$ & Nominal (centro educativo / institución social) \\
\hline Calificación del alumnado en las prácticas del curso anterior & $\mathrm{VI}$ & Nominal (suspenso, aprobado, notable, sobresaliente, matrícula de honor) \\
\hline
\end{tabular}

\section{Identificación de variables (tutoría académica)}

La prueba de igualdad de Levene determinó que la varianza de la VD 1 era igual entre los grupos intersujetos: $L(72,178)=1.322$; sig. $=0.71$, cumpliendo el supuesto de homocedasticidad. ANOVA determinó que el modelo propuesto era significativo en la variable intragrupo, $F(72,250)=1.479$, sig. $=0.020$. El modelo propuesto con las VI utilizadas explica el $12.10 \%$ de la varianza de la VD 1. La Tabla 5 muestra también aquellas VI que son significativas y que afectan a la satisfacción del alumnado. Se observa cómo sólo la variable género afecta significativamente a las percepciones de satisfacción del alumnado sobre su tutor/a académico (sig.=0.023). Específicamente en esta variable que es significativa, el sexo femenino mantiene percepciones superiores $(M=3.30)$ respecto al sexo masculino $(M=2.90)$.

Tabla 5: ANOVA: satisfacción del alumnado sobre la figura del tutor/a académico $\left(R^{2}=.374 ; R^{2}\right.$ ajustada $\left.=.121\right)$

\begin{tabular}{|l|l|l|l|l|l|}
\hline Origen & Tipo III de suma de cuadrados & gl & Cuadrático promedio & F & Sig. \\
\hline Modelo corregido & $90.835^{\mathrm{a}}$ & 72 & 1.262 & 1.479 & 0.020 \\
\hline Género & 4.508 & 1 & 4.508 & 5.284 & 0.023 \\
\hline Acceso a la universidad & 0.085 & 1 & 0.085 & 0.100 & 0.753 \\
\hline Grado Educativo & 4.912 & 3 & 1.637 & 1.919 & 0.128 \\
\hline Calificación previa & 7.196 & 4 & 1.799 & 2.109 & 0.082 \\
\hline Lugar de las practicas & 1.438 & 1 & 1.438 & 1.686 & 0.196 \\
\hline Error & 151.835 & 178 & 0.853 & & \\
\hline Total & 3097.500 & 251 & & & \\
\hline Total corregido & 242.670 & 250 & & & \\
\hline
\end{tabular}




\section{Identificación de variables (tutor/a profesional)}

El test de Levene violó el supuesto de homocedasticidad asumiendo que la varianza de la VD 2 era diferente entre los grupos inter-sujetos, por lo que se utilizó un contraste por Bonferroni, $L(72,176)=1.786$; sig. $=0.001$. ANOVA determinó que el modelo propuesto era significativo en la variable intragrupo, $F(72$, $248)=2.100$, sig. $=0.001$. El modelo propuesto con las VI utilizadas explica el $24.20 \%$ de la varianza de la VD 2. La Tabla 6 muestra aquellas VI que son significativas y que afectan a la satisfacción del alumnado sobre su tutor/a profesional.

Tabla 6: ANOVA: satisfacción del alumnado sobre la figura del tutor/a profesional $\left(R^{2}=.462 ; R^{2}\right.$ ajustada $\left.=.242\right)$

\begin{tabular}{|l|l|l|l|l|l|}
\hline Origen & Tipo III de suma de cuadrados & gl & Cuadrático promedio & F & Sig. \\
\hline Modelo corregido & $111.671^{\mathrm{a}}$ & 72 & 1.551 & 2.100 & 0.001 \\
\hline Género & 0.782 & 1 & 0.782 & 1.058 & 0.017 \\
\hline Acceso a la universidad & 11.040 & 1 & 11.040 & 14.946 & 0.001 \\
\hline Grado Educativo & 9.091 & 3 & 3.030 & 4.102 & 0.008 \\
\hline Calificación previa & 2.694 & 4 & 0.673 & 0.912 & 0.458 \\
\hline Lugar de las practicas & 4.231 & 1 & 4.231 & 5.728 & 0.018 \\
\hline Error & 130.006 & 176 & 0.739 & & \\
\hline Total & 3809.593 & 249 & & & \\
\hline Total corregido & 241.677 & 248 & & & \\
\hline
\end{tabular}

Respecto al género, se encontraron diferencias significativas en la satisfacción del alumnado sobre su tutor/a profesional (sig.=0.017). Concretamente, el alumnado femenino $(M=3.83)$ percibió una satisfacción mayor que el alumnado masculino $(\mathrm{M}=3.43)$. Respecto a la satisfacción del alumnado en función del acceso al sistema universitario que tuvieron, el alumnado que accedió a través de las pruebas de selectividad obtuvo una mayor satisfacción $(M=3.94)$ respecto al que utilizó otros medios de acceso $(M=3.28)$, existiendo diferencias significativas entre ambas distribuciones (sig.=0.001). También se encontraron diferencias significativas en función del grado educativo en el que realizaba las prácticas el alumnado (sig. $=0.008$ ). Específicamente, el alumnado que percibió una mayor satisfacción se corresponde a los del grado de Educación Infantil $(M=3.94)$, seguido de Pedagogía $(M=3.84)$, Educación Primaria $(M=3.57)$ y Educación Social $(M=3.21)$. Por último, el lugar donde se llevaron a cabo las prácticas también afecta a la satisfacción del alumnado respecto a su tutor/a profesional (sig.=0.018). Concretamente, el alumnado que cursó las prácticas en centros educativos percibió un mayor nivel de satisfacción con su tutor/a profesional $(M=3.83)$ respecto al alumnado que realizó las prácticas en instituciones sociales $(M=3.49)$.

\section{DISCUSIÓN FINAL}

En esta investigación, se ha pretendido conocer el nivel de satisfacción del alumnado que está realizando su último periodo de formación práctica para la obtención del Grado en las titulaciones vinculadas a la Facultad de Ciencias de la Educación (Andalucía, España). Los resultados se corroboran con los obtenidos por Cid et al. (2011), López et al. (2012), García et al. (2018), Pinto (2019) y Vallejo et al. (2019), puesto que, en todos estos estudios, al igual que en el presente trabajo, el nivel de satisfacción de los estudiantes en relación a la tutorización tanto del tutor/a académico como del tutor/a profesional eran elevados. No coincidiendo con Valverde (2016) debido a que dicho autor en un estudio realizado con una muestra de 2001 estudiantes concluyó que los estudiantes estaban muy satisfechos con las labores del tutor/a profesional, pero no tanto con el tutor/a académico. Por otro lado, según el alumnado es necesario una mayor comunicación y coordinación entre ambos tutores/as, resultados que coinciden con los obtenidos por Melesse (2014), Izadinia (2016), Nguyen (2017), McGraw \& Davis (2017), Maddamsehi (2018), Ruffinelli et al. (2020), siendo necesarios programas sólidos de formación permanente para los tutores/as, tal y como afirma García et al. (2018), así como espacios permanentes de reflexión interinstitucional (Sánchez et al., 2011) donde se analicen en profundidad las competencias y roles de cada uno de los agentes implicados (Helleve \& Ulvik, 2019), para que no se devalúe la función tutorial tanto académica como profesional (Liesa y Vived, 2010).

En relación al género, el nivel de satisfacción respecto a la tutorización tanto académica como profesional es mayor en las mujeres, datos que contrastan con los obtenidos por Vallejo et al. (2019) puesto que ellos no encontraron diferencias significativas en función del género. Es el alumnado que estudia el Grado de Educación Infantil, ha accedido a la universidad mediante Pruebas de Acceso Universitarias y que realizan las prácticas en centros educativos los que están más satisfechos con la labor del tutor/a profesional. Esta situación puede ser debida a que dicho alumnado, al no haber tenido prácticas previas en su formación académica, no poseen un punto de contraste. 
A la luz de los resultados, se puede afirmar, por tanto, que una adecuada tutorización influye de manera decisiva en la satisfacción y en el desarrollo de las competencias profesionales del alumnado. Se pone de manifiesto, en definitiva, la necesidad de optimizar el proceso de tutorización durante las prácticas, lo cual lleva a reconsiderar la valoración que se hace de las prácticas externas en los planes de estudio actuales y la labor de coordinación que se viene realizando entre los tutores/as profesionales y académicos.

\section{CONCLUSIONES}

Las principales conclusiones de este estudio son; 1) es necesario desde las instituciones de educación superior dedicar más esfuerzos políticos y económicos a la formación de los tutore/as académicos y profesionales; 2) es necesario incluir vías sólidas de coordinación y comunicación existente ambos, donde se analicen en profundidad sus roles; 3) se debe considerar como algo muy positivo la consolidación de una red de tutores/as profesionales, que asuman en sus respectivos centros, el compromiso de la formación práctica del alumnado; 4) debe haber un plan de prácticas en el que el trabajo tutorial que se realice sea útil no solo para el alumnado, sino también para los profesionales implicados y las propias organizaciones; en definitiva; y 5) para que el alumnado adquiera un verdadero desarrollo profesional, es necesaria una estrecha vinculación entre todos los organismos y agentes implicados en el proceso formativo.

\section{REFERENCIAS}

Alonso-García, S., Rodríguez-García, A. M., y Cáceres-Reche, M., Análisis de la Acción Tutorial y su Incidencia en el Desarrollo Integral del Alumnado. El Caso de la Universidad de Castilla La Mancha, España, doi: 10.4067/S071850062018000300063, Formación universitaria, 11(3), 63-72 (2018)

Casillas, S., Cabezas, M., y Serrate, S., Evaluación de competencias: Retos en la Formación Práctica de los Pedagogos, Meta: Avaliaçao, 9 (25), 90-109 (2017).

Chan-Pavon, M., Mena-Romero, D., Escalante-Euán, J., y Rodríguez-Martín, M., Contribución de las Prácticas Profesionales en la formación de los Estudiantes de la Facultad de Ingeniería Química de la Universidad Autónoma de Yucatán (México), doi: 10.4067/S0718-50062018000100053, Formación universitaria, 11(1), 53-62 (2018)

Cid, A., Pérez, A., y Sarmiento, J.A., La tutoría en el Practicum. Revisión de la literatura, Revista de Educación, 354, 127-154 (2011).

García, J.R., Mena Lorenzo, J., y Conill, J., La superación del Profesor tutor de 2018 los Estudiantes de Formación Pedagógica durante sus Prácticas Laborales, MENDIVE, 16(1), 5-19 (2018)

Cramer, S. F., Giving back: Mentoring others as you were mentored, College \& University, 91 (4), $37-40$ (2016).

Gairín, J., Díaz, A., del Arco, I., y Flores, O., Efecto e impacto de las Prácticas Curriculares de los Grados de Educación Infantil y Primaria: la perspectiva de estudiantes, tutores y coordinadores, Educación XX1, 22(2), 17-43 (2019)

González, X. A., y Hevia, I., El Practicum de la Licenciatura de Pedagogía: Estudio empírico desde la perspectiva del alumnado, Revista de Educación, 354, 209-236 (2011)

Helleve, I., y Ulvik, M., Tutors seen through the eyes of Mentors assumptions for participation in third space in Eeacher Education, doi: 10.1080/02619768.2019.1570495, European Journal of Teacher Education, 42:2, 228-242 (2019)

Holtz, P., y Gnambs, T., The improvement of student teachers' instructional quality during a 15- week field experience: a latent multimethod change analysis, doi: 10.1007/s10734-016-0071-3, Higher Education, 74(4), 669-685 (2017)

Izadinia, M., Student Teachers' and mentor Teachers' perceptions and expectations of a mentoring relationship: do they match or clash?. Professional Development in Education, 42(3), 387-402 (2016)

López, E., Valentín, A., y González, M., De las condiciones del Practicum de la licenciatura en Psicología a las competencias del Grado: análisis de las valoraciones de los alumnos, Aportaciones recientes al estudio de la Motivación y las Emociones, pp. 47-55, Fénix Editora, Sevilla, España (2012)

Liesa, M., y Vived, E., El nuevo Practicum del grado de magisterio. Aportaciones de alumnos y profesores, Revista Estudios sobre Educación, 18, 201-228 (2010)

Maddamsetti, J., Perceptions of Pre-service Teachers on mentor Teachers' roles in promoting Inclusive Practicum: case studies in US Elementary School contexts, doi: 10.1080/02607476.2017.1422608, Journal of Education for Teaching, 44(2), 232-236 (2018)

Martínez, M. E., y Raposo, M., Funciones generales de la tutoría en el Practicum: entre la realidad y el deseo en el desempeño de la acción tutorial, Revista de Educación, 354, 155-181 (2011)

Martínez, R., Ruiz, R., y Valladares, L., Innovación en la Educación Superior: hacia las sociedades del conocimiento, Fondo de Cultura Económica, México DF (2012)

Martins, M., Costa, J., y Onofre, M., Practicum experiences as sources of pre-service teachers' self-efficacy, doi: 10.1080/02619768.2014.968705, European Journal of Teacher Education, 38(2), 263-279 (2015) 
Mayorga, M. J., Sepúlveda, M.P., Madrid, D., y Gallardo, M., Grado de satisfacción y utilidad profesional de las prácticas externas del alumnado de la Facultad de Ciencias de la Educación de la Universidad de Málaga (España). Revista Perfiles Educativos, 39 (157), 140-159 (2017)

McGraw, A., y Davis, R., Mentoring for Pre-service Teachers and the use of inquiry-oriented feedback, doi: 10.1108/IJMCE-03-2016-0023, International Journal of Mentoring and Coaching in Education, 6(1), 50-63 (2017)

Melesse, T., Assessment on the Implementation of the Pre-service Practicum Program in Teacher Education Colleges (Dessie College of Teacher Education in Focus), Assessment, 5(20), 97-110 (2014)

Nicol, D., Thomson, A., y Breslin, C., Rethinking Feedback Practices in Higher Education: A Peer Review Perspective, doi: 10.1080/02602938.2013.795518, Assessment \& Evaluation in Higher Education 39: 102-122 (2014)

Nguyen, H. T. M., TESOL Practicum Mentoring for Pre-service Teachers: The Vision Versus the Reality, In Models of Mentoring in Language Teacher Education, pp. 47-70, Springer International Publishing, Switzeland (2017)

Olmedo, E. O., Gómez, G. C., y López, E., Design an Action Plan to Structure a Competency-Based Curriculum: a Study in Spanish University System, doi: 10.18562/ijee.026, Journal of Educational Excellence, 3(2), 14-34 (2016)

Overton, D., Findings and implications of the relationship of pre-service educators, their university tutor and in-service teachers regarding professional development in science in the primary school system, doi: 10.1080/19415257.2017.1388268, Professional Development in Education, 44(5), 595-606 (2018).

Pinto-Llorente, A. M., Pre-Service English Teachers' Perception of the Practicum Experience, doi: 10.4018/978-1-52259228-0.ch013 In English Language Teaching in a Post-Method Paradigm, pp. 302-318, IGI Global, England (2019).

Rodriguez-Loera, R., y Onrubia, J., La percepción de Estudiantes de Maestro de último semestre sobre la relación entre teoría y práctica en el Prácticum, doi: 10.37042/practicum.2019.4.2.3, Revista Prácticum, 4(2), 42-59 (2019)

Ruffinelli, A., de la Hoz, S., y Álvarez, C., Practicum tutorials in initial Teacher training: conditions, strategies, and effects of reflective practice, doi: 10.1080/14623943.2019.1708712, Reflective Practice, 21:1, 54-67 (2020)

Sánchez, C. A., Ramírez, S., y García, A., La formación de tutores externos de Practicum en educación: Diseño, desarrollo y evaluación de una experiencia, Revista de Docencia Universitaria, 9 (2), 119-145 (2011)

Sulistiyo, U., Mukminin, A., Abdurrahman, K., y Haryanto, E., Learning to Teach: A Case Study of Student Teachers' Practicum and Policy Recommendations, The Qualitative Report, 22(3), 712-731 (2017)

Teng, F., Emotional Development and Construction of Teacher Identity: Narrative interactions about the Pre-service Teachers' Prácticum Experiences, doi: 10.14221/ajte.2017v42n11.8, Australian Journal of Teacher Education, 42(11), 117-134 (2017)

Vallejo, A. P., Estrella, A. C., y López-Barajas, D. M., Perceived student satisfaction and professional relevance in the Practicum, Profesorado, Revista de Currículum y Formación del Profesorado, 23(1), 365-392 (2019)

Valverde, M. A., Las Prácticas externas extracurriculares: tutorización y seguimiento como medida para incrementar la calidad, Revista Practicum, 1 (1), 54-79 (2016)

Vicente, M., Educación y Trabajo en Ciencias de la Educación: Aportes desde el Estudio de las Trayectorias Profesionales de sus Graduados, 10.4067/S0718-50062012000600006, Formación universitaria, 5(6), 51-62 (2012)

Yangin, G., y Gungor, M. N., Exploring the Use of Narratives to Understand Pre-service Teachers' Prácticum Experiences from a Sociocultural Perspective, doi: 10.14221/ajte.2018v43n4.9, Australian Journal of Teacher Education, 43(4), 159-174 (2018).

Yuan, E. R., The dark side of mentoring on pre-service language teachers' identity formation. Teaching and Teacher Education, 55, 188-197 (2016) 